Research Article

\title{
Energy Control Strategy for Parallel Hybrid Electric Vehicle Based on Terminal Neural Network
}

\author{
Haitao Yan (i) ${ }^{1,2}$ and Yongzhi $\mathrm{Xu}^{2}$ \\ ${ }^{1}$ School of Mechanical and Precision Instrument Engineering, Xi'an University of Technology, Xi'an, Shaanxi 710048, China \\ ${ }^{2}$ Sanmenxia Polytechnic, Sanmenxia, Henan 472000, China \\ Correspondence should be addressed to Haitao Yan; yht0211@stu.xaut.edu.cn
}

Received 13 October 2021; Revised 25 October 2021; Accepted 29 October 2021; Published 17 November 2021

Academic Editor: Bai Yuan Ding

Copyright (c) 2021 Haitao Yan and Yongzhi Xu. This is an open access article distributed under the Creative Commons Attribution License, which permits unrestricted use, distribution, and reproduction in any medium, provided the original work is properly cited.

\begin{abstract}
Energy control strategy is a key technology of hybrid electric vehicle, and its control effect directly affects the overall performance of the vehicle. The current control strategy has some shortcomings such as poor adaptability and poor real-time performance. Therefore, a transient energy control strategy based on terminal neural network is proposed. Firstly, based on the definition of instantaneous control strategy, the equivalent fuel consumption of power battery was calculated, and the objective function of the minimum instantaneous equivalent fuel consumption control strategy was established. Then, for solving the time-varying nonlinear equations used to control the torque output, a terminal recursive neural network calculation method using BARRIER functions is designed. The convergence characteristic is analyzed according to the activation function graph, and then the stability of the model is analyzed and the time efficiency of the error converging to zero is deduced. Using ADVISOR software, the hybrid power system model is simulated under two typical operating conditions. Simulation results show that the hybrid electric vehicle using the proposed instantaneous energy control strategy can not only ensure fuel economy but also shorten the control reaction time and effectively improve the real-time performance.
\end{abstract}

\section{Introduction}

Energy crisis and environmental pollution are two major problems that need to be solved in today's social development. With the development of society, environmental pollution has become increasingly prominent, especially the recent haze weather, seriously affecting people's daily life and even threatening life and health, and automobile exhaust emission is one of the main sources of this severe weather. Traditional fuel vehicles have been difficult to meet increasingly demanding energy saving standards and environmental protection indicators, while new energy vehicles such as fuel cell vehicles have encountered bottlenecks due to constraints of technology, cost, or infrastructure [1-5]. In addition, the power battery technology of pure electric vehicle is not mature enough to meet the requirements of long-distance continuous driving. In contrast, hybrid electric vehicle technology is advancing steadily.
From the current situation, it is necessary and feasible to take hybrid electric vehicle as a transitional model to relieve energy and environmental pressure.

As hybrid electric vehicles have two energy sources, engine and battery, it is particularly important to make reasonable use of the two energy sources to provide the vehicle with good fuel economy and emission performance [6-9]. Therefore, the energy control of the vehicle becomes a crucial part of the hybrid power system. Hybrid electric vehicles can be divided into series, parallel, and hybrid according to the type, quantity, and connection relationship of parts [10-13]. Parallel hybrid power system can reduce fuel consumption and pollutant emission of the vehicle without greatly increasing the cost and the complexity of the vehicle structure. Therefore, the research focus of this paper is on parallel hybrid electric vehicle (HEV).

Energy control strategy refers to the torque distribution and coordination control strategy between the engine and 
motor. At present, there are four basic types of energy control strategies [14,15]: (1) logic threshold control strategy; (2) instantaneous control strategy; (3) global optimal control strategy; (4) fuzzy control strategy. The full name of instantaneous control strategy is instantaneous equivalent minimum fuel consumption control strategy. In each control cycle of the hybrid power system, the optimal power output of the engine and motor is calculated with the goal of obtaining the lowest fuel consumption. Instantaneous control strategies can also combine fuel economy and emission performance. Instantaneous control strategy can achieve the minimum equivalent fuel consumption within each control cycle, but it requires a lot of calculation and is difficult to achieve. In addition, according to the optimization theory, the sum of minimum values is not equal to the minimum value of sum, so the instantaneous control strategy cannot achieve global optimization. The global optimal control strategy [16] can theoretically obtain the lowest fuel consumption in the real sense, but the outstanding disadvantage is that the driving condition of the vehicle must be known during the whole operation process, which is obviously inconsistent with the reality. At present, this control strategy can only be used to evaluate other control effects, and it is difficult to be applied in real-time vehicle control.

\section{Literature Review}

In view of the problems of the above control strategies, many scholars have improved and optimized the energy control strategy of HEV by using different research methods.

In the existing research results, Yin et al. [17] introduced the condition recognition technology into the fuzzy control strategy to solve the shortcoming that it could not adapt to different working conditions and expand the application scope of fuzzy control. However, the paper did not give a clear conclusion about which working conditions it was applicable to. Han et al. [18] used the stochastic dynamic programming theory to solve the problem that the global optimal control strategy requires to know future driving conditions in advance. The simulation results show that the control method can achieve good results in a variety of driving conditions, but the calculation is large and the simulation time is long. Calafiore et al. [19] proposed a stochastic model predictive control method to try to solve the problem that future driving conditions of hybrid electric vehicles are difficult to predict. Ming et al. [20] established an intelligent control strategy of hybrid electric vehicle considering the influence of driving conditions by using fuzzy neural network. The actual vehicle verification results show that the established control strategy reduces the influence of driving condition fluctuation on the performance of electric vehicles. However, this method takes too long to obtain recognition results. These research results have improved the existing energy control strategies to a certain extent and improved the performance of hybrid power system, but so far there is no optimal control scheme that can be used for practical control. The optimization and improvement of $\mathrm{HEV}$ energy control strategy need further research.
The terminal neural network is a kind of neural network model with bounded convergence error in finite time. The effectiveness of the network solving model usually includes two aspects, that is, higher solving accuracy of the model (for example, in the field of chaotic system control) and faster solving speed (for example, in the field of terminal sliding mode control). The finite-time convergent dynamic characteristics of the terminal state greatly improve the convergence efficiency of the dynamic system and, at the same time, obtain higher system convergence accuracy. Chai et al. [21] proposed a class of terminal system with dynamic convergence, which has the dynamic property of finite-time convergence and was applied to the solution of time-varying equations and the repetitive motion planning of redundant manipulator, both of which achieved high convergence accuracy. Different from the finite-time convergent dynamic network, the finite-state convergent neural network proposed by Yuan et al. [22] can adopt any form of nonlinear activation function, which is applicable to a wider range and more in line with actual needs.

Therefore, a terminal neural network model for controlling torque output is established in this paper. Firstly, the objective function of the instantaneous control strategy is defined, and the nonlinear equations used to control the torque output are solved by using terminal neural network models. Then, an example is given based on the neural network model, and the simulation results are obtained. The simulation results show that the terminal network controller can achieve good fuel economy and improve the real-time control performance.

\section{Principle of Instantaneous Control Strategy}

3.1. Transient Control Policy Definition. The full name of instantaneous control strategy is instantaneous equivalent fuel consumption minimum control strategy [23,24], so it can be seen that this control rule mainly includes two aspects:

(1) Equivalent fuel consumption: for a general hybrid electric vehicle, its power battery electric energy needs to be maintained within a certain range, that is, the power battery electric energy consumed in a certain stage (except the recovered braking energy) needs to be supplemented by a certain amount of fuel consumed by the engine in the future operation of the hybrid electric system. Therefore, an equivalence relationship between the energy consumed by the power battery and the fuel required to replenish it is needed.

(2) Instantaneous optimization: in each control period, according to the power requirements of the hybrid power system, the optimal power output of the motor and engine is calculated in real time with the minimum equivalent fuel consumption as the standard, and the two are controlled based on this rule.

The minimum control strategy of instantaneous equivalent fuel consumption of HEV can be simply expressed as 


$$
M=\sum \operatorname{Min}\left\{m_{f c}\left[T_{f c}(t), \omega_{f c}(t)\right] \Delta t+m_{m c_{-} e q}\left[T_{m c}(t), \omega_{m c}(t)\right] \Delta t\right\} .
$$

At the same time, the following constraints should also be met:

$$
\begin{aligned}
& 0<T_{f c}(t)<T_{f c-\max }, \\
& 0<\omega_{m c}(t)<\omega_{m c-\max }, \\
& S O C_{\text {final }}-S O C_{\text {initial }}=0,
\end{aligned}
$$

where $\omega_{f c}$ is the engine speed, $\omega_{m c}$ is the motor speed, $\omega_{m c_{-} \max }$ is the maximum motor speed, $T_{f_{\mathcal{C}_{-} \max }}$ is the maximum engine torque, $m_{f c}$ is the engine fuel consumption at every instant, $m_{m c_{-} e q}$ is the equivalent fuel consumption of power battery, $T_{f c}$ is the engine torque, $T_{m c}$ the is motor torque, $\mathrm{SOC}_{\text {initial }}$ is the initial state of charge of power battery, and $\mathrm{SOC}_{\text {final }}$ is the state of charge at the end of power battery.

\subsection{Calculation Method of Equivalent Fuel Consumption of} Power Battery. This paper establishes the relationship between power battery and fuel energy consumed by engine. Specific methods are as follows:

(1) Establish the relationship between engine fuel consumption rate and motor output torque:

$$
b=f\left(T_{m c}\right)
$$

When the total demand torque is known, the relation curve between the motor output torque and engine fuel consumption rate can be established according to engine universal characteristic curve. As shown in Figure 1, points A, B, C, and D correspond to the working conditions of engine driving alone and charging for power battery, engine driving alone and not charging for power battery, engine and motor codriving vehicle, and motor driving alone.

(2) Establish the corresponding relationship between power battery variation and motor output torque:

The qualitative relation curve between the variation of power battery and the output torque of the motor is shown in Figure 2, where three points A, B, and C, respectively, represent the working conditions in which the engine drives the motor to charge the power battery, the engine drives the system without the power battery participating in the operation, and the engine and the power battery jointly drive the hybrid power system.

According to the relation curve between the state of charge and voltage of the power battery and the relation curve between the current of the motor and the output torque, the power battery variation corresponding to the output torque of each motor can be calculated, and the relation between the variation of the power battery and the output torque of the motor can be established.

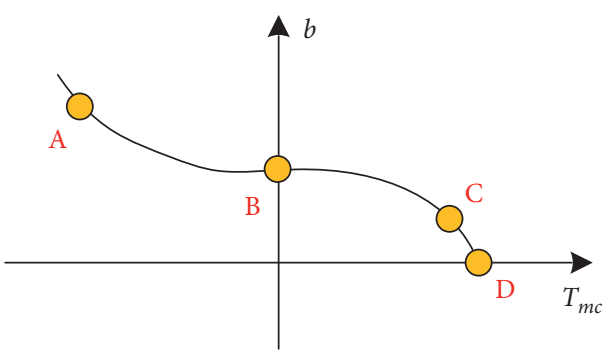

Figure 1: Qualitative relation curve between engine fuel consumption rate and motor output torque.

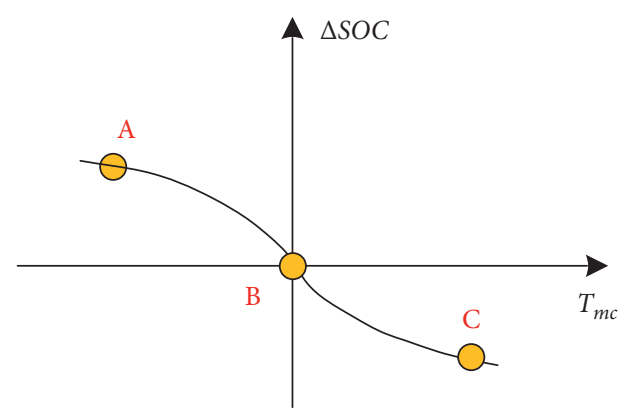

FIGURE 2: Qualitative relation curve between power battery variation and motor output torque.

$$
\Delta \mathrm{SOC}=f\left(T_{m c}\right)
$$

(3) Establish the corresponding relationship between engine fuel consumption rate and actual variation of power battery:

$$
b=f\left(\Delta S O C_{\text {act }}\right)
$$

where $\triangle S O C_{a c t}$ is the actual change of the power battery.

(4) Calculate the fuel energy corresponding to the actual change of power battery:

According to the relationship between the actual change of power battery obtained in Step 3 and the engine fuel consumption rate, the corresponding relationship between the actual change of power battery and the fuel energy consumed by the engine can be established, as shown in Figure 3.

The above method makes use of the equal relationship between the electric energy consumed by the power battery and the electric energy replenished by the power battery to establish the relationship between the actual change of the power battery and the fuel energy consumed by the engine. The disadvantage of this approach is that it does not take into account that the operating state of the hybrid system is changing from moment to moment. The above method is based on the assumption that the demand speed of vehicles at the future moment is the same as that at present, which is contrary to the actual situation. Based on the analysis of the energy flow of parallel hybrid electric vehicle, the instantaneous control strategy is calculated. 


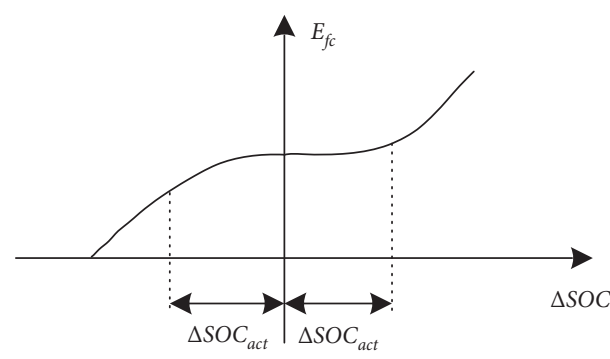

FIGURE 3: Qualitative relation curve between actual variation of power battery and engine energy consumption.

\subsection{Calculation of Instantaneous Equivalent Fuel Con-} sumption of Power Battery. The working mechanism of the instantaneous control strategy can be briefly described as follows: in order to achieve the lowest fuel consumption of the normal running of the vehicle, the distribution ratio of the power required by the vehicle between the engine and the motor is optimized in real time in each control cycle. Therefore, a transient optimal objective function which can accurately reflect the actual operation characteristics of $\mathrm{HEV}$ should be established. The energy flow diagram of the hybrid power system is shown in Figure 4.

The engine power, the power allocated by the engine to the motor for charging the power battery, and the total required power of the system satisfy the following relational expression:

$$
P_{f c N}=P_{f c Y}-P_{m c_{-} \text {chg }}=P_{\text {req }},
$$

where $P_{\text {req }}$ is the driving power required by the vehicle at a certain time, $P_{m c_{-} \text {chg }}$ is the charging power of the motor, and $P_{f c Y}$ is the engine power.

Therefore, the fuel consumption rate of the engine charging the power battery at a certain time in the future can be expressed by the fuel consumption rate when the engine only participates in driving the vehicle. The equivalent fuel consumption is

$$
m_{m c-q q}=\frac{b_{\text {chg }} P_{m c-\text { chg }}}{\eta_{m c} \eta_{\text {chg }} \eta_{\text {dischg }}},
$$

where $b_{\text {chg }}$ is the fuel consumption rate of the engine for charging the power battery, $\eta_{m c}$ is the motor efficiency, $\eta_{\text {chg }}$ is the charging efficiency of the power battery, and $\eta_{\text {dischg }}$ is the discharging efficiency of the power battery.

3.4. Objective Function of Instantaneous Control Strategy. According to the objective function of the minimum control strategy for instantaneous fuel consumption given previously, equation (1) can be rewritten as

$$
\begin{aligned}
& M= \sum \operatorname{Min}\left\{m_{f c}\left[P_{f c}(t)\right] \Delta t\right. \\
&\left.+m_{m c_{-} q}\left[P_{m c}(t)\right] \Delta t\right\}, \\
& m_{m c_{-} e q}\left[P_{m c}(t)\right]=f_{\text {eq_dischg }} P_{m c} .
\end{aligned}
$$

In order to explain the different functions of the motor at different times in the process of deriving instantaneous fuel consumption, charge and discharge can be unified when the objective function is expressed. Therefore, equation (8) can be rewritten as

$$
M=\sum \operatorname{Min}\left\{m_{f c}\left[P_{f_{c}}(t)\right] \Delta t+\left[\lambda f_{\text {eq_dischg }}+(1-\lambda) f_{\text {eq_chg }}\right] P_{m c} \Delta t\right\},
$$

where $\lambda=\left[1+\operatorname{sign}\left(T_{m c}\right)\right] / 2$.

According to equation (10), the control effect of the instantaneous control strategy depends on the size of the two variables. These two variables are respectively called the equivalent fuel consumption coefficient of the power battery under the discharge condition and the charging condition, and their specific values are determined by the hybrid electric vehicle configuration, efficiency characteristics, and driving conditions.

\section{Instantaneous Control Strategy Based on Terminal Neural Network}

The control rule of instantaneous control strategy can be regarded as the nonlinear mapping of single output, and the power battery output refers to the motor output torque. Consider the following dynamic characteristic equation to construct the recursive neural network model [25]:

$$
\dot{E}=-\rho S(E)
$$

where $E$ is the $n \times n$ dimensional error matrix, $\rho>0$ is the adjustable gain coefficient, and $S(E)$ is the activation function, which is strictly monotonically increasing odd function.

It is known that the recursive neural network is asymptotically stable by the properties of activation function. The asymptotic convergence performance is analyzed below. According to equation (11),

$$
\begin{gathered}
\frac{d E}{d t}=-\rho E \Longrightarrow \frac{1}{E} d E=-\rho d t, \\
\int_{E_{0}}^{E} \frac{1}{E} \mathrm{~d} E=\int_{0}^{t}-\rho \mathrm{d} t \Longrightarrow \ln E-\ln E_{0}=-\rho t,
\end{gathered}
$$

where $E_{0}$ is a constant. According to equation (13), when $E=0, t \longrightarrow \infty$, so the error converges to zero in infinite time; the recursive neural network model characterized by the dynamic characteristics of asymptotic stability is called asymptotic convergence network.

In order to realize the finite-time convergence of energy control of parallel hybrid electric vehicles, the control rules of instantaneous control strategy are regarded as the solution of time-varying nonlinear equations of single output (motor output torque), and the optimization objective function is equation (12), which can be expressed as follows:

$$
\left\{\begin{array}{c}
f_{1}\left(x_{1}, x_{2}, \ldots, x_{n}\right)=0 \\
f_{2}\left(x_{1}, x_{2}, \ldots, x_{n}\right)=0 \\
\vdots \\
f_{n}\left(x_{1}, x_{2}, \ldots, x_{n}\right)=0
\end{array}\right.
$$




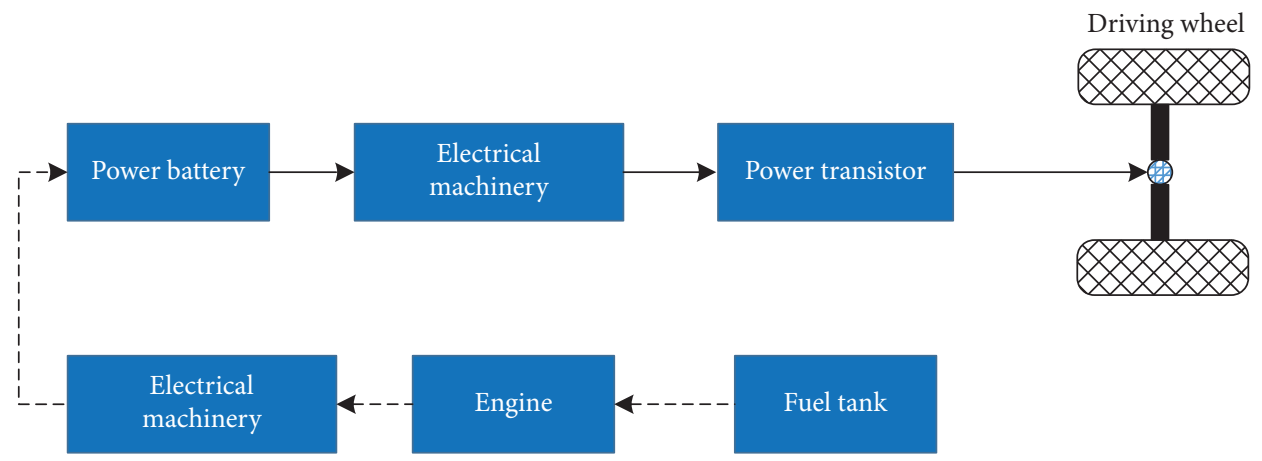

Figure 4: Energy flow diagram of hybrid power system.

where $x_{n}$ is the $n$-th unknown variable and $f_{n}$ is a smooth differentiable nonlinear function.

To solve the nonlinear equations, the error equation is defined as follows:

$$
e(t)=f(x)-0 .
$$

The time needed to obtain the solution of nonlinear equations by using the terminal neural network is finite, but the asymptotic network needs enough time. In order to simplify the analysis, the time-varying nonlinear equations are set as follows:

$$
\left\{\begin{array}{l}
\frac{\ln x_{1}(t)-1}{(t-1)=0} \\
1(t) x_{2}(t)-\exp \left(\frac{1}{(t+1)}\right) \sin (t)=0 \\
x_{1}^{2}(t)-\sin (t) x_{2}(t)+x_{3}(t)-2=0 \\
x_{1}^{2}(t)+x_{2}^{2}(t) x_{3}(t)+x_{4}(t)-t=0 .
\end{array}\right.
$$

Then, the test calculation result is

$$
X^{*}(t)=\left[\begin{array}{c}
x_{1}^{*} \\
x_{2}^{*} \\
x_{3}^{*} \\
x_{4}^{*}
\end{array}\right]=\left[\begin{array}{c}
\exp \left(\frac{1}{(t+1)}\right) \\
\sin (t) \\
2-\exp \left(\frac{2}{(t+1)}\right)+\sin (t) \\
t-2-2 \sin ^{2}(t)
\end{array}\right] .
$$

The initial value can be any value, and $\mathbf{X}(0)=\left[\begin{array}{llll}1 & 1 & 1 & 1\end{array}\right]^{\mathrm{T}}$ in this paper.

A recursive neural network model is constructed to solve the time-varying nonlinear equations. The convergence characteristics are analyzed according to the activation function graph, and then the stability of the model is analyzed respectively and the time of error convergence to zero is deduced.

\section{Terminal Neural Network Calculation}

The terminal neural network based on BARRIER function is used to calculate the solution of nonlinear equations. The neural network equation is as follows:

$$
\dot{e}=-\varepsilon \operatorname{Bar}(e, \delta) \text {. }
$$

BARRIER excitation function [26] is

$$
S(e)=\operatorname{Bar}(e, \delta)=\sqrt{\frac{|e|}{|e|+1 / \delta}} \operatorname{sgn}(e) .
$$

The parameters in the terminal neural network are $\varepsilon=5$ and $\delta=0.5$. The calculation results are shown in Figure 5, and the calculate value $X(t)$ converges to the ideal value $\mathbf{X}^{*}(t)$.

Figure 6 shows the comparison between the two norms of $f(x)$ in the terminal network and the asymptotic network. It can be seen from the figure that $\|f(x)\|_{2}$ obtained by using the terminal network has a faster convergence speed and converges to zero in finite time.

\section{Simulation Experiment and Result Analysis}

6.1. Simulation Software and Parameters. In this paper, the hybrid electric vehicle simulation software ADVISOR is used as a test platform to test whether the neural network controller can improve the response speed of instantaneous control. ADVISOR, which stands for advanced vehicle simulator, was developed by the U.S. National Renewable Energy Laboratory. ADVISOR adopts modular programming idea and MATLAB/Simulink programming language, and the code is completely free to the public so that it has good secondary development and cosimulation with other software functions. The simulation process of ADVISOR is shown in Figure 7. First, the actual speed and fuel consumption rate of the vehicle are calculated according to the model data and component data, and then simulation experiments are carried out. Finally, simulation graphics and data are output.

In order to increase the authenticity of the simulation experiment, the parameters of the vehicle in this simulation experiment were formulated according to the data of a listed hybrid model. The main parameters are shown in Table 1. 


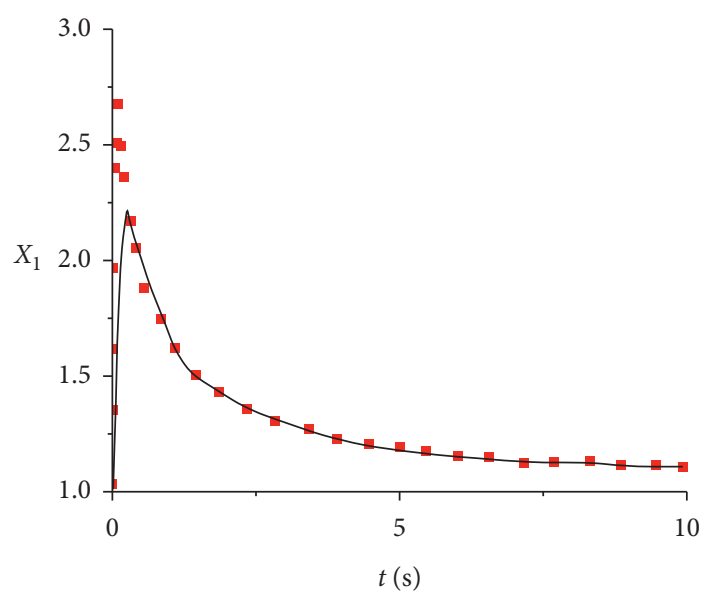

- True value

- Computed value

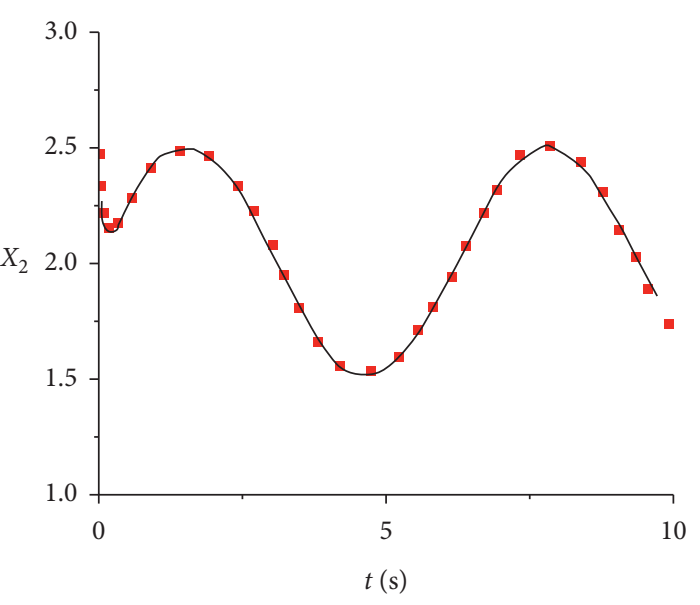

- True value

- Computed value

Figure 5: Comparison between the true result and resultant values of nonlinear equations.

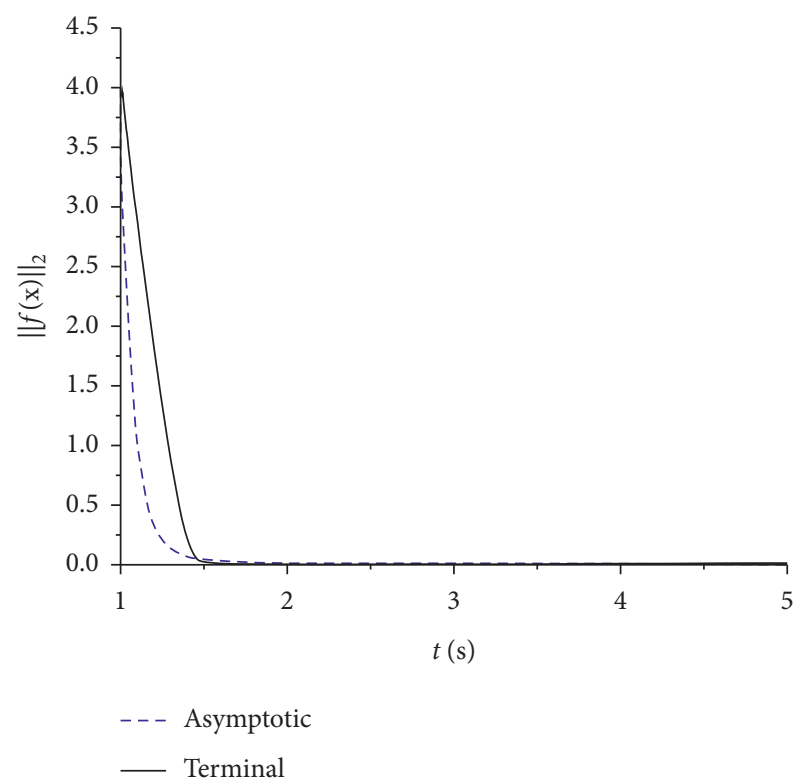

Figure 6: Comparison of $\|f(x)\|_{2}$ under terminal network and asymptotic network.

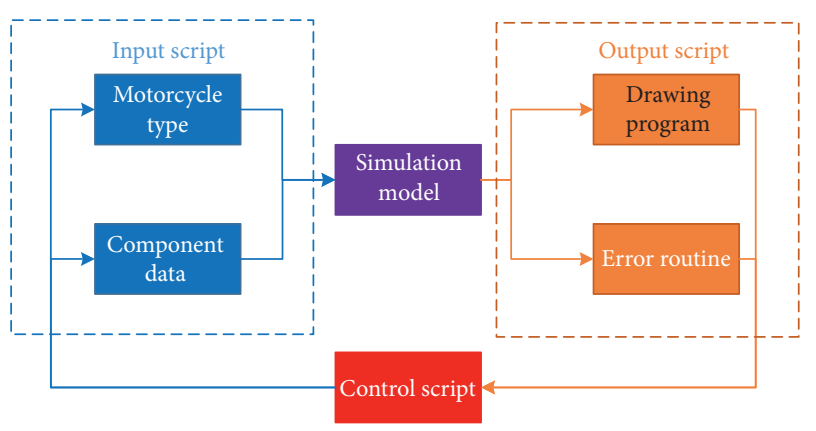

FIGURE 7: ADVISOR simulation process.
The choice of driving conditions will have a great impact on the performance of the test vehicle. The new European Urban Driving Cycle (NEDC) and Highway Fuel Economy Test (HWFET) were selected to carry out simulation tests. Table 2 shows the relevant parameters of the two commonly used driving conditions.

6.2. Comparison and Analysis of Simulation Results. After all the simulation work is completed, the simulation results of traditional instantaneous control, fuzzy neural network control, and terminal neural network control are compared. The three control strategies are compared in SOC, fuel consumption, and simulation time.

In Figure 8, NEDC_mdf, NEDC_fnn, and NEDC_tnn, respectively, represent the simulation results corresponding to the three schemes of traditional instantaneous control, fuzzy neural network control, and terminal neural network control of the hybrid electric vehicle model under NEDC conditions. HWFET_mdf, HWFET_fnn, and HWFET_tnn, respectively, represent the simulation results corresponding to the traditional instantaneous control, fuzzy neural network control, and terminal neural network control schemes of the hybrid electric vehicle model under HWFET conditions. As can be seen from Figure 8, the terminal neural network controller can approximately simulate the traditional instantaneous control rules, and the SOC value is maintained in the high efficiency range.

As can be seen from Table 3, the terminal neural network controller realizes the advantages of low fuel consumption of the fuzzy neural network control strategy and can bring good economy to the vehicle. As can be seen from Table 4, the simulation time of the terminal neural controller is significantly shortened compared with the other two control strategies, thus improving the real-time performance of the control. 
TABLE 1: Parameters of HEV for simulation.

\begin{tabular}{lcc}
\hline Automobile key module & Parameter & Value \\
\hline Full vehicle & Mass & $1605 \mathrm{~kg}$ \\
& http://dict.youdao.com/w/drag\%20coefficient/ & 0.32 \\
\hline http://dict.youdao.com/w/engine/ & http://dict.youdao.com/w/displacement/ & $2.5 \mathrm{~L}$ \\
& http://dict.youdao.com/w/maximum\%20power/ & $118 \mathrm{~kW}$ \\
http://dict.youdao.com/w/battery\%20pack/ & Type & Hydrogen ballast \\
& Number of modules & 34 \\
\hline Motor & Capacity & 6.5 Ah \\
& http://dict.youdao.com/w/nominal\%20voltage/ & $244.8 / 650 \mathrm{~V}$ \\
\hline
\end{tabular}

TABLe 2: Two typical driving conditions.

\begin{tabular}{lcc}
\hline Parameter & NEDC & HWFET \\
\hline Cycle time $(\mathrm{s})$ & 1184 & 765 \\
Maximum speed $\left(\mathrm{km} \cdot \mathrm{h}^{-1}\right)$ & 10.93 & 16.51 \\
http://dict.youdao.com/w/operating\%20range/ $(\mathrm{km})$ & 120 & 96.4 \\
Average velocity $\left(\mathrm{km} \cdot \mathrm{h}^{-1}\right)$ & 33.21 & 77.58 \\
http://dict.youdao.com/w/maximum\%20acceleration/ $\left(\mathrm{m} \cdot \mathrm{s}^{-2}\right)$ & 1.06 & 1.43 \\
Maximum deceleration $\left(\mathrm{m} \cdot \mathrm{s}^{-2}\right)$ & -1.39 & -1.48 \\
Idle time $(\mathrm{s})$ & 298 & 6 \\
Number of stopping & 13 & 1 \\
\hline
\end{tabular}

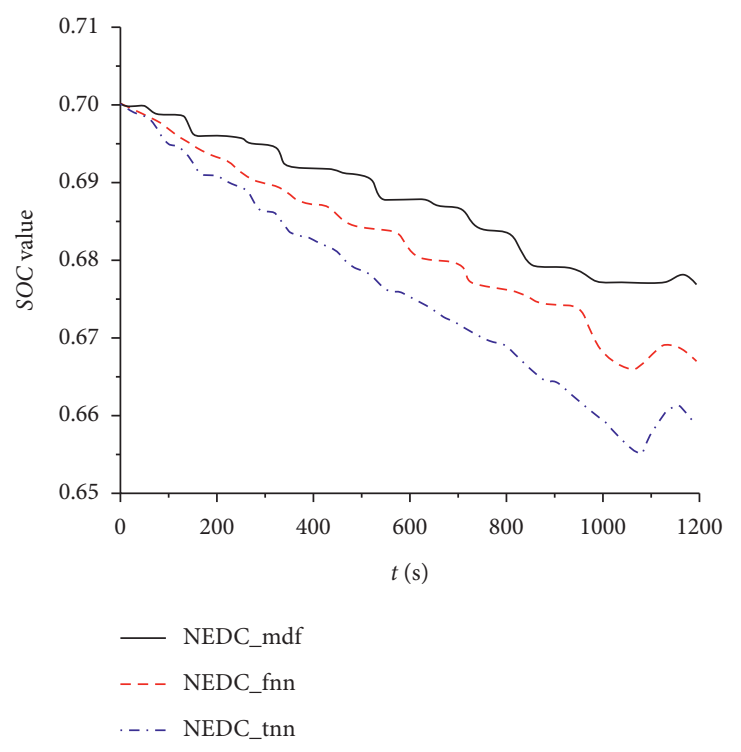

(a)

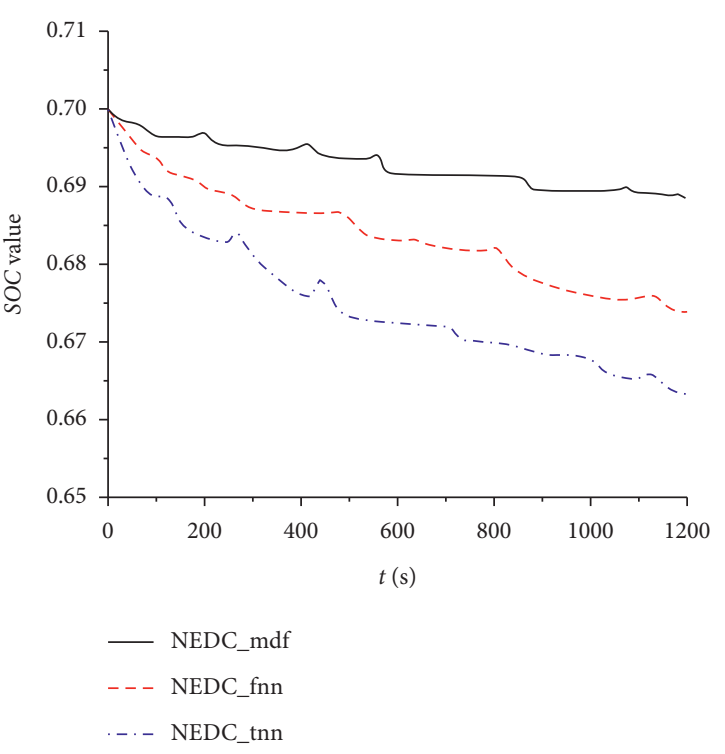

(b)

Figure 8: Comparison of power battery SOC. (a) NEDC. (b) HWFET.

TABle 3: Fuel consumption per 100 kilometers.

\begin{tabular}{lccr}
\hline & Conventional instantaneous control & Fuzzy neural network control & Terminal neural network control \\
\hline NEDC $\left(\mathrm{L} \cdot 100 \mathrm{~km}^{-1}\right)$ & 10.1 & 9.8 & 9.8 \\
HWFET $\left(\mathrm{L} \cdot 100 \mathrm{~km}^{-1}\right)$ & 9.2 & 7.8 & 8.0 \\
\hline
\end{tabular}


TABLE 4: Simulation time.

\begin{tabular}{lccc}
\hline & Conventional instantaneous control & Fuzzy neural network control & Terminal neural network control \\
\hline NEDC (s) & 45.6 & 102.3 & 15.6 \\
HWFET (s) & 35.2 & 89.4 & 11.3 \\
\hline
\end{tabular}

\section{Conclusions}

In view of the shortcomings of traditional instantaneous control strategy, such as slow response and weak real-time performance, this paper uses neural network controller as torque control device to seek for more reasonable control of engine and motor output power of parallel strong hybrid electric vehicle. Under two typical operating conditions, ADVISOR software was used to simulate the hybrid electric vehicle model embedded with traditional instantaneous control, fuzzy neural network control, and terminal neural network control, respectively. The results show that the reaction time of the instantaneous control can be greatly reduced by the control strategy of the terminal neural network under the premise of ensuring the fuel economy and emission performance of the vehicle [27-30].

\section{Data Availability}

The experimental data used to support the findings of this study are available from the corresponding author upon request.

\section{Conflicts of Interest}

The authors declare that they have no conflicts of interest to report regarding the present study.

\section{References}

[1] S. Zhang, R. Xiong, and F. Sun, "Model predictive control for power management in a plug-in hybrid electric vehicle with a hybrid energy storage system," Applied Energy, vol. 185, pp. 1654-1662, 2017.

[2] J. Cao and R. Xiong, "Reinforcement learning-based real-time energy management for plug-in hybrid electric vehicle with hybrid energy storage system," Energy Procedia, vol. 142, pp. 1896-1901, 2017.

[3] S. Suganya, S. Charles Raja, and P. Venkatesh, "Smart management of distinct plug-in hybrid electric vehicle charging stations considering mobility pattern and site characteristics," International Journal of Energy Research, vol. 41, no. 14, pp. 2268-2281, 2017.

[4] M. G. Arend and T. Franke, "The role of interaction patterns with hybrid electric vehicle eco-features for drivers' ecodriving performance," Human Factors: The Journal of the Human Factors and Ergonomics Society, vol. 59, no. 2, pp. 314-327, 2017.

[5] A. Cerofolini, "Optimal supervisory control strategy for a transmission-mounted electric drive hybrid electric vehicle," International Journal of Automotive Technology, vol. 20, no. 4, pp. 663-677, 2019.

[6] C. Xiang, F. Ding, W. Wang, and W. He, "Energy management of a dual-mode power-split hybrid electric vehicle based on velocity prediction and nonlinear model predictive control," Applied Energy, vol. 189, no. 5, pp. 640-653, 2017.

[7] C.-M. Lai, Y.-H. Cheng, M.-H. Hsieh, and Y.-C. Lin, "Development of a bidirectional DC/DC converter with dualbattery energy storage for hybrid electric vehicle system," IEEE Transactions on Vehicular Technology, vol. 67, no. 2, pp. 1036-1052, 2018.

[8] Z. D. Asher, D. A. Baker, and T. H. Bradley, "Prediction error applied to hybrid electric vehicle optimal fuel economy," IEEE Transactions on Control Systems Technology, vol. 26, no. 6, pp. 1-14, 2017.

[9] X. Zhou, D. Qin, and J. Hu, "Multi-objective optimization design and performance evaluation for plug-in hybrid electric vehicle powertrains," Applied Energy, vol. 208, no. 12, pp. 1608-1625, 2017.

[10] Y. Chao, X. Jiao, L. Liang, Z. Yuanbo, and C. Zheng, “A robust Ho control-based hierarchical mode transition control system for plug-in hybrid electric vehicle," Mechanical Systems and Signal Processing, vol. 99, no. 6, pp. 326-344, 2018.

[11] R. Koubaa and L. Krichen, "Double layer metaheuristic based energy management strategy for a Fuel Cell/Ultra-Capacitor hybrid electric vehicle," Energy, vol. 133, no. 8, pp. 1079-1093, 2017.

[12] S. Ahmadi, S. M. T. Bathaee, and A. H. Hosseinpour, "Improving fuel economy and performance of a fuel-cell hybrid electric vehicle (fuel-cell, battery, and ultra-capacitor) using optimized energy management strategy," Energy Conversion and Management, vol. 160, no. 5, pp. 74-84, 2018.

[13] B. Duan, Q. Wang, X. Zeng, Y. Gong, D. Song, and J. Wang, "Calibration methodology for energy management system of a plug-in hybrid electric vehicle," Energy Conversion and Management, vol. 136, pp. 240-248, 2017.

[14] Z. Zhao, D. Lei, J. Chen, and H. Li, "Optimal control of mode transition for four-wheel-drive hybrid electric vehicle with dry dual-clutch transmission," Mechanical Systems and Signal Processing, vol. 105, pp. 68-89, 2018.

[15] M. Kohli, L. M. Prevedello, R. W. Filice, and J. R. Geis, "Implementing machine learning in radiology practice and research," American Journal of Roentgenology, vol. 208, no. 4, pp. 1-7, 2017.

[16] Z. Lei, D. Sun, Y. Liu et al., "Analysis and coordinated control of mode transition and shifting for a full hybrid electric vehicle based on dual clutch transmissions," Mechanism and Machine Theory, vol. 114, pp. 125-140, 2017.

[17] X.-x. Yin, Y.-g. Lin, W. Li, Y. J. Gu, H. W. Liu, and P. F. Lei, “A novel fuzzy integral sliding mode current control strategy for maximizing wind power extraction and eliminating voltage harmonics," Energy, vol. 85, pp. 677-686, 2015.

[18] L. Bai, J. Guo, X. Tianyou, and Y. Minghai, "Emotional Monitoring of Learners Based on EEG Signal Recognition," Procedia Computer Science, vol. 174, pp. 364-368, 2020.

[19] G. Han, X. Pu, Z. He, and C. Liu, "Integrated planning and allocation: a stochastic dynamic programming approach in container transportation," Chaos, Solitons \& Fractals, vol. 114, pp. 264-274, 2018. 
[20] G. C. Calafiore and L. Fagiano, "Stochastic model predictive control of LPV systems via scenario optimization," Automatica, vol. 49, no. 6, pp. 1861-1866, 2013.

[21] L. Ming, Y. Ying, L. Liang, L. Yao, and W. Zhou, "Energy management strategy of a plug-in parallel hybrid electric vehicle using fuzzy control," Energy Procedia, vol. 105, pp. 2660-2665, 2017.

[22] J. Chai, J.-E. Guo, L. Meng, and S.-Y. Wang, "Exploring the core factors and its dynamic effects on oil price: an application on path analysis and BVAR-TVP model," Energy Policy, vol. 39, no. 12, pp. 8022-8036, 2011.

[23] Z. Yuan and L. Yuan, "Existence and global convergence of periodic solution of delayed neural networks," Mathematical and Computer Modelling, vol. 48, no. 1, pp. 101-113, 2017.

[24] J. C. Juinne-Ching Liao and S. N. Sheng-Nian Yeh, "A novel instantaneous power control strategy and analytic model for integrated rectifier/inverter systems," IEEE Transactions on Power Electronics, vol. 15, no. 6, pp. 996-1006, 2000.

[25] K. Furukawa, H. M. Tarafdar, T. Ise, and S. Kumagai, "A control strategy for active filters using quasi-instantaneous positive sequence extraction filters," Electrical Engineering in Japan, vol. 148, no. 1, pp. 54-65, 2010.

[26] L. Wu, Y. Wang, X. Li, and J. Gao, "Deep attention-based spatially recursive networks for fine-grained visual recognition," IEEE Transactions on Cybernetics, vol. 49, pp. 1791-1802, 2018.

[27] W. Bai, B. Zhang, Q. Zhou, and R. Lu, "Multigradient recursive reinforcement learning NN control for affine nonlinear systems with unmodeled dynamics," International Journal of Robust and Nonlinear Control, vol. 30, no. 8, pp. 17-29, 2019.

[28] H. Fathabadi, "Fuel cell hybrid electric vehicle (FCHEV): novel fuel cell/SC hybrid power generation system," Energy Conversion and Management, vol. 156, no. 6, pp. 192-201, 2018.

[29] T. K. Nizami and A. Chakravarty, "Neural network integrated adaptive backstepping control of DC-DC boost converter," IFAC-PapersOnLine, vol. 53, no. 1, pp. 549-554, 2020.

[30] K. Khnissi, C. B. Jabeur, and H. Seddik, "A smart mobile robot commands predictor using recursive neural network," Robotics and Autonomous Systems, vol. 131, pp. 84-90, 2020. 